Meta

Journal des traducteurs

Translators' Journal

\title{
Terminologie du cinéma
}

\section{Jean Giraud}

Volume 12, numéro 4, décembre 1967

Aspects de la terminologie

URI : https://id.erudit.org/iderudit/002919ar

DOI : https://doi.org/10.7202/002919ar

Aller au sommaire du numéro

Éditeur(s)

Les Presses de l'Université de Montréal

ISSN

0026-0452 (imprimé)

1492-1421 (numérique)

Découvrir la revue

Citer cet article

Giraud, J. (1967). Terminologie du cinéma. Meta, 12(4), 118-124.

https://doi.org/10.7202/002919ar

Ce document est protégé par la loi sur le droit d'auteur. L'utilisation des services d'Érudit (y compris la reproduction) est assujettie à sa politique d'utilisation que vous pouvez consulter en ligne.

https://apropos.erudit.org/fr/usagers/politique-dutilisation/
Cet article est diffusé et préservé par Érudit.

Érudit est un consortium interuniversitaire sans but lucratif composé de l’Université de Montréal, l'Université Laval et l'Université du Québec à Montréal. Il a pour mission la promotion et la valorisation de la recherche. https://www.erudit.org/fr/ 


\section{rerminologie dur cinnéma}

Quelle place revient, dans la langue du cinéma, au vocabulaire proprement technique? Pour essayer d'y voir clair, un bref rappel historique semble opportun.

Remontons donc aux débuts, c'est-à-dire aux vingt-cinq années environ qui conduisent du moment où le cinéma va être inventé (ultime phase d'une longue chaîne industrieuse de recherches) jusqu'à celui où la première guerre mondiale penche vers son terme. On n'ose parler encore de terminologie, car celle-ci existe à peine en tant qu'aspect d'un phénomène linguistique plus vaste. La raison? Le fait linguistique lui-même, fort maigrelet, va son petit bonhomme de chemin. Le contexte manque. Entendons par là un genre de production comme de spectacle impatient de rompre les cloisons artisanales; un public sensibilisé en profondeur; des moyens de l'atteindre, de le convaincre, d'élever ses exigences autrement que par l'hyperbole d'une réclame assez puérile; enfin, une spécialisation professionnelle: le «cinématographiste» primitif est un Maître Jacques; il a le «bonimenteur » pour compère. Ajoutons qu'une information internationale sommaire encourage peu le souci de la nomenclature. En somme, l'empirisme règne sur presque toute la ligne. Songeons qu'à la fin de 1905 - dix ans après les premières séances publiques - l'avoir total ne dépasse pas quatre-vingts mots, soit la moitić environ des noms de jouets et d'appareils qui constituaient le vocabulaire du précinéma, de 1825 à 1895. Et la plupart sont cmpruntés à la photographie, aux projections fixes, à l'illusionnisme, au journalisme, à la mécanique, à la chimie, au théâtre: exemple typique du besoin qu'éprouve un art naissant de s'appuyer sur le «patrimoine * proche ou lointain.

Cependant, quelques termes de base: réaliser, producteur, diriger, adaptation, scénariste, panoramique, montage, studio, apparaissent, parfois bien timidement, à partir de 1906. Puis, tout à coup, avec l'entrée en guerre des États-Unis, le film américain commence à déferler sur nos écrans, avec une première vague de néologismes qu'une partie au moins du public va s'habitucr à lire et à entendre. Car cette période de l'épanouissement et de la fin du cinéma muet s'outille en vue 
d'une compréhension interne du "savoir-filmer ». Pour répandre les dénominations nouvelles il existe, désormais, non seulement des monographies et des magazines à bon marché - souvent férus d'un snobisme aussi appuyé que facile - mais un mouvement d'avant-garde, une légion de jeunes Turcs. Leurs armes et munitions: livres, numéros spéciaux de revues, chroniques de presse enfin sérieuses sinon toujours impartiales, à l'intention du public actif et remuant qui se presse dans les ciné-clubs éclos au Quartier latin. Pour la première fois certains professionnels du cinéma - on commence à dire cinéastes — prennent contact avec des cinéphiles qui, à tue-tête, se proclament tels.

De cette terminologie déployée sur douze ou treize années, retenons en particulier ces emprunts qui seront adoptés (avec ou sans guillemets), francisés ou traduits:

- Métiers, spécialités du cinéma: producer, director, manager, supervisor, scriptgirl, star, starlet, vamp, stunt, bathing beauties, gagman, cast.

- Préconstruction et construction du film: synopsis, continuity, close shot, long(-j) shot, flash, flash(-)back, gag.

- Genres de films: thriller, western, serial, story picture, moving picture, sound picture, talking picture (talkie).

- Contact avec le public: preview, fan.

- Procédés, recherches: technicolor.

- Aspect financier: block-booking, block-system.

D'autre part naissent ces essais de «grammaires » et «syntaxes» du cinéma qui voudront être, aussi bien pour le spectateur évolué que pour le cinéaste amateur ou apprenti, des ouvrages clés d'initiation technique, petits traités ou recueils de définitions exemplifiées et illustrées, permettant de suivre la chaîne d'opérations qui jalonnent la genèse et la naissance d'un film. Valeur linguistique faible; valeur technique inégale (inévitables redites, copiages et bâclages), réelle cependant, car la nomenclature essentielle est analysée et diffusée ${ }^{1}$.

Le cinéma a passé avec succès son certificat d'aptitude professionnelle. Son avoir linguistique atteint, vers 1930 , un millier de créations, néologismes de polysémie et emprunts. Mais que ce nombre ne fasse pas trop illusion. Si l'on ôte les archaïsmes déjà nombreux, les victimes de la collision sémantique, les mots burlesques, les hapax et tout ce que les grilles de l'usage ont promptement arrêté, le régiment devient compagnie quant à la vie réelle du mot, à sa circulation, à son assimilation, à sa condition adulte, à son «passeport ». Telle est la loi de la nature: le lexique propose, le locuteur dispose. Il semble bien, toutefois, que la nomenclature technique ait moins souffert de cet effet de déperdition que le vocabulaire général ou appréciatif. Celui-ci est sujet aux caprices de la mode, aux mauvaises comme aux bonnes fortunes de la néologie. Celle-là, au contraire, évolue sous la seule emprise du besoin, de la recherche et du perfectionnement. Elle va du concept à la chose, au geste, à l'initiative, à la trouvaille, à la procédure. Elle résout des problèmes petits ou grands. Un mot n'est effacé par un autre que si cet autre apporte

1. Jean Mitry a, en quelque sorte, perfectionné ces moyens de vulgarisation grâce à son documentaire Ecrire un film, réalisé il y a environ dix ans. 
un mieux. Et les traditions du métier sont plus vivaces que les broderies charmantes de la stylistique. Ainsi, les expressions familières que manie le peuple laborieux et ingénieux des studios - mécaniciens, électriciens, accessoiristes, ouvriers divers n'ont fait que se multiplier, même si l'objet a depuis longtemps disparu (le fameux 《tour de manivelle»). Cet aspect de la langue écrite et surtout parlée du cinéma, puis de la télévision, mérite sympathie.

Avec le film parlant, l'industrialisation de la couleur, le relief, l'électronique, la stéréophonie, les écrans spéciaux ou circulaires, la miniaturisation de certains matériels et la marche vers le «cinéma total», nous voici dans l'âge scientifique hautement spécialisé. La langue ne peut qu'en recevoir une impulsion décisive. L'avoir lexical réel du cinéma a largement doublé depuis $1930^{2}$. Ses caractères antérieurs se sont élargis et accentués. La distinction entre la terminologie et les autres modes de l'extension sémantique est aujourd'hui plus nette. Essayons de brosser le parallèle.

Quelques observations, d'abord, sur le vocabulaire que déterminent l'usage et la mode. Il s'est relativement peu enrichi en mots importants: hollywoodien, présence, suspense, pin-up, play-back, néo-réalisme, caméra-stylo, cinéma direct, distanciation, générique, cinéma d'essai ... Encore plusieurs d'entre eux passent-ils par la technique. Ainsi, on ne confondra pas: «la mise en scène », expression ancienne empruntée au théâtre, avec "une mise en scène », long métrage à intrigue, par opposition au court ou moyen métrage documentaire ou «d'essai ». C'est la grande étape dans la carrière d'un jeune réalisateur, sa véritable entrée dans le théâtre d'opérations.

Le vocabulaire analytique, critique et filmographique a fructifié par quatre voies essentielles:

- Persistance, avec des hauts et des bas, du goût de l'emprunt. Quelques italianismes, germanismes, russismes, etc., se mêlent à l'américain et au cher « franglais ».

- Appel déraisonnable et désordonné aux affixes, notamment aux suffixes -ien et -esque, dont les valeurs sont souvent confondues, tant ces modes de formation sont appliqués, à tort et à travers et sans discrimination aucune, à des éphèbes débutants comme aux Grands du cinéma.

- Surenchères du farfelu, du baroque, du méprisant ou du railleur, de l'ingénieux, du jeu de mots laborieux, du prétentieux, du facile. Les critiques-enfants s'amusent. Ce vocabulaire fait sourire ou irrite, puis il s'oublie. C'est le petit magasin de curiosités, et parfois le sottisier du lexique ${ }^{3}$.

- Fait beaucoup plus marquant, enfin: de très nombreux écrivains de cinéma puisent maintenant, tantôt avec mesure et tantôt jusqu'à satiété, dans le récent

2. D'après François Le Lionnais, président de l'Association des écrivains scientifiques de France, "il se crée un millier de mots par jour dans chaque langue d'un pays civilisé " (sous bénéfice d'inventaire).

3. Cf. J. Giraud, "Contribution au vocabulaire farfelu du cinéma ", Vie et langage, octobre 1965; février, juin et décembre 1966; mars 1967. 
vocabulaire philosophique, psychologique, sociologique, ethnographique, voire mathématique et pédagogique ${ }^{4}$; à moins qu'ils ne préfèrent créer, à l'image de ces langues spéciales, les termes qui leur font défaut. Le mouvement filmologique, lancé à Paris en 1946 par une constellation d'universitaires, de spécialistes et de techniciens, n'a pas médiocrement contribué à cette sorte d'osmose où s'intellectualise - sans éviter les stéréotypes ni le jargon — une façon d'écrire naguère beaucoup plus fermée à d'autres influences que celles des arts traditionnels ${ }^{5}$.

Et ceci nous ramène à la technique. Les vocabulaires que l'on forge dans toutes les branches des sciences humaines et expérimentales ne sont-ils pas, en effet, autant de terminologies, «boîtes à outils » de l'expression?

Quelques exemples permettront de mieux comparer à ce qui précède les caractères et les tendances de la langue technique des cinéastes d'aujourd'hui, devenue en grande partie, on le sait, celle des «téléastes 》 du petit écran:

1. Georges Sadoul intitule deux de ses chroniques sur "Le langage du film ${ }^{6}$ : «Doit-on cinescoper un cinémascope? ? et «Faut-il « ampexer » ou « magnétographier?». Ces quatre néologismes appellent des remarques nombreuses et caractéristiques. Les deux premiers recréent, ou presque, des dénominations qui remontent aux origines: kinescope, cinématoscope, sans oublier le très vieux kinématoscope de Sellers (1861). Du reste, cinescope( $r$ ) francise le rugueux «kinescope », qu'il a remplacé dans les dictionnaires comme plus seyant à notre goût. Et ni l'Office français du vocabulaire, ni le général Leschi, ni les Académiciens siégeant au Conseil supérieur de la R.T.F. (devenue l'O.R.T.F.) ne furent étrangers à cette substitution de lettre. D'autre part, l'Office a condamné «Ampex», simple nom de marque, au profit de magnétoscope, désignation scientifique de l'appareil.

Nous eûmes, jadis, le vitagraphe et le vitascope, le kinétographe et le kinétoscope, parmi tant d'autres monstres et pachydermes morphologiques; faut-il dire maintenant: magnétographe et magnétographier, ou magnétoscope et magnétoscoper? Je ne m'engagerai pas, aujourd'hui, dans ces querelles. La langue ancienne ou moderne du cinéma n'a cessé d'accrocher à d'innombrables locomotives les racines presque jumelles (ou sœurs rivales) graphein et skopein. Les formations disparaissent de l'usage avec les appareils périmés, puis ressurgissent sous des graphies à peine différentes, car le jeu des combinaisons n'est pas illimité. Cette terminologie fut et demeure une jungle où le «super» et le « rama» cherchent de nos jours à faire la loi.

Ce qu'il faut retenir des questions posées par Georges Sadoul, en les survolant un peu, c'est que la technique crée et recrée sans cesse, quitte à revenir à ses premières amours linguistiques. Et ces créations n'échappent plus au contrôle, à l'examen critique, aux essais de normalisation d'organismes compétents; et les dictionnaires mettent plus de hâte que dans le passé à enregistrer les néologismes

4. Cf. notamment les Cahiers du cinéma (1967), ainsi que Cinéma 67 (et années antérieures).

5. Cf. J. Girand, "Les arcanes de la filmologie », Vie et langage, août 1965.

6. Les Lettres françaises, 21 février et 24 avril 1963. 
essentiels. Faits nouveaux que justifie l'inquiétude du linguiste devant le foisonnement des termes techniques. Contester, débattre, choisir, devient indispensable. Souvenons-nous des protestations qu'à juste titre souleva le barbarisme «mondiovision » surgi sous la plume de journalistes ignares ou trop pressés pour réfléchir une seule seconde (c'est évidemment ou mondovision ou mondialvision que l'on peut dire).

2. Passons des appareils au tournage, et du mot complexe au mot simple et bref, que les racines grecques ne bardent pas.

Plan et séquence, expressions éprouvées par l'usage puisqu'elles sont entrées dans la langue du cinéma respectivement vers 1912 et vers 1925 , ont longtemps servi à désigner deux composantes bien différenciées, par leur valeur-durée, de la construction filmique. La première (comme l'anglais shot) «cadre * (to shoot) une scène, un personnage ou un détail à un certain échelon d'éloignement ou de proximité, durant un temps variable mais le plus souvent court ou très court. La deuxième "enferme» une suite d'actions montrées à divers plans: notre œil ne reste pas inactif, notre regard se déplace, diraient les « grammairiens 》 de l'écran, devant une fraction de l'espace où s'accomplit quelque chose. Le rapport entre ces termes était donc de contenu à contenant, de fragment discontinu à continuité, comme le paragraphe dans l'épisode romanesque ou la réplique dans la scène de théâtre.

De plan/séquence à plan-séquence, la ponctuation modifie tout. Ici la terminologie évolue parce qu'elle reflète de nouvelles façons de filmer, d'écrire pour l'œil. La technique sert une stylistique. Ce qu'est le plan-séquence, André Bazin, Alexandre Astruc, Louis Malle, Marcel Bluwal (pour la télévision) et beaucoup d'autres nous l'apprennent; un plan long, étiré, conduisant d'une seule haleine une phase de l'action dont la durée peut atteindre trois ou quatre minutes. Une manière de pont suspendu, hardiesse de l'architecture filmique. Les positions de la caméra suivent les déplacements des personnages, dans un cadre fixe mais dont la * profondeur du champ » souligne la valeur spatiale. Une rythmique souple, des panoramiques fréquents découpent dans cette immobilité apparente des secteurs tour à tour «privilégiés », diraient les Gestaltistes. Ainsi le langage de l'image, dans les mutations que lui imposent de nouveaux modes de travail devant et derrière la caméra, trouve une deuxième source d'enrichissement pour la terminologie, sans que celle-ci soit bouleversée, puisqu'il s'agit simplement de mettre à jour un chapitre de la « grammaire » ou de la «syntaxe».

3. C'est d'extension sémantique qu'il faut maintenant parler à propos d'un mot qui a fait fortune: audio(-)visuel. Apparu aux États-Unis vers 1940 (aides audiovisuelles à l'instruction) puis répandu en France et en Europe, dans l'immédiat après-guerre, en même temps que mass-media et communications de masse, il ne s'applique encore, en 1951, d'après le Vocabulaire de la psychologie de Piéron qu'aux «projections fixes ou mobiles [films] commentées et expliquées par le maître ». Mais, dès 1955, 1'I.D.H.E.C. énumère, comme "moyens 》 de la « méthode » audio-visuelle: "le cinéma, la télévision, les projections fixes, les disques, les tests psychologiques ». S'y sont ajoutés la radio, le magnétophone, père du 
laboratoire de langues, et certains matériels employés à la formation professionnelle dans l'enseignement et dans l'industrie, ainsi qu'aux campagnes d'alphabétisation: bloc-papier, tableau de feutre, tableau magnétique. Ainsi le qualificatif audio-visuel a-t-il revêtu un sens de plus en plus global, puisqu'il comprend des instruments techniques de caractère unisensoriel mais les associe entre eux. L'appellation primitive ne correspond plus très bien à une réalité devenue très élastique.

C'est pourquoi l'on a voulu lancer une expression certainement meilleure: celle de médiateurs techniques (technical media en anglo-américain, Technische Mittler en allemand). Dans ce complexe, le cinéma demeure comme l'élément fondamental et emblématique: la recherche du «synchronisme » ne fut-elle pas, dès la période artisanale, son ambition d'abord maladroite ? Même les possibilités auditives et visuelles, dont disposait l'industrie américaine d'il y a trente ans, sont largement dépassées. La revue française «à la page » dans les divers domaines de la science théorique et appliquée, du reportage, de l'information et du vocabulaire technique, c'est Interaudiovision ${ }^{\top}$. À notre avis, ce titre exprime à lui seul, beaucoup mieux que «moyens audio-visuels », le stade auquel sont parvenus, et la finition de l'outillage, et la portée des échanges entre spécialistes.

Ainsi, dans toutes les branches de son immense activité, la terminologie actuelle manifeste deux tendances principales: d'une part, un déploiement et un enrichissement continus non moins qu'irréversibles; d'autre part, la force d'impulsion exercée par notre «vieux » cinéma qui, d'emprunteur à ses débuts, se fait prêteur: les langues spéciales de la télévision et de la radio lui doivent beaucoup, celle du disque et de la magnétophonie quelque peu. On pourrait encore rappeler qu'il a créé une petite nomenclature propre au film d'animation, lequel déborde lui-même la notion, déjà ancienne, de dessin animé: «cinéma bis », autre technique. Mais arrêtons-nous là.

Constatons ce signe de maturité: le néologisme n'est pas toujours lancé à la légère, ni accepté sans réflexion ni discussion. La langue, estime justement $\mathrm{M}$. Jean Darbelnet, est « un instrument d'analyse, un vocabulaire, un classeur». En outre, cette fructification technique paraît user de procédés plus sérieux, mieux soudés à la réalité, moins mécanistes que peuvent l'être la suffixation massive des noms propres ou le culte du farfelu (faits linguistiques, l'un encombrant, l'autre amusant, mais tous deux de faible signification pour la langue, car ils usent trop de « recettes $\gg$ ).

Sans doute cette affirmation de puissance s'accompagne-t-elle des risques auxquels n'est pas demeuré indifférent le «Colloque sur la terminologie scientifique et technique », qui s'est tenu à Montréal en octobre 1966 sous les auspices de l'Office de la langue française du Québec ${ }^{8}$. Ce sont, notamment, la longueur et la pesanteur du mot; son manque de transparence d'une langue à l'autre; le calque

7. Revue des techniques audio-visuelles et de leurs applications, Paris, Editions techniques spécialisées.

8. Je remercie M. Robert Dubuc de m'en avoir aimablement adressé le compte rendu, d'où est extraite la citation de Jean Darbelnet. 
morphologique maladroit; l'intrication du technique pur et de la finalité publicitaire; la difficulté de certaines dérivations ${ }^{9}$; enfin, le problème important au Canada et même en France, où l'abus des emprunts peut devenir source d'obscurité. Mais il a si vivement retenu l'attention du Comité linguistique de Radio-Canada, que ce m'est un agréable devoir de rendre hommage à nos amis québecois pour les nombreuses fiches de cinéma, radio, télévision et autres «médiateurs techniques », déjà publiées par leurs soins.

Cette langue vit intensément et souvent, il faut le dire, intelligemment, grâce aux efforts conjugués des chercheurs, des techniciens, des cinéastes créateurs et des linguistes. Mais cette «constellation » n'est qu'au commencement de ses peines. Du moins peut-on espérer la fondation prochaine d'un corps de spécialistes, d'un comité international des terminologies, qui serait à la fois carrefour, tribunal d'arbitrage et organe de sélection et de mise au point - je n'ai pas dit de « fixation » pure et simple, notion absurde. C'est sur ce vœu que je terminerai.

JEAN GIRAUD

9. Interaudiovision est-il suffixable ? 